\title{
ZUPT-aided INS Bypassing Stance Phase Detection by Using Foot-Instability-Based Adaptive Covariance
}

This paper was downloaded from TechRxiv (https://www.techrxiv.org).

\section{LICENSE}

CC BY 4.0

SUBMISSION DATE / POSTED DATE

28-07-2021 / 02-08-2021

\section{CITATION}

Jao, Chi-Shih; Shkel, Andrei M. (2021): ZUPT-aided INS Bypassing Stance Phase Detection by Using FootInstability-Based Adaptive Covariance. TechRxiv. Preprint. https://doi.org/10.36227/techrxiv.15063099.v1

DOI

10.36227/techrxiv.15063099.v1 


\title{
ZUPT-aided INS Bypassing Stance Phase Detection by Using Foot-Instability-Based Adaptive Covariance
}

\author{
Chi-Shih Jao, Student Member, IEEE, and Andrei M. Shkel, Fellow, IEEE
}

\begin{abstract}
In this paper, we propose a Foot-Instability-Based Adaptive (FIBA) covariance to dynamically adjust the covariance matrix for the pseudo-zero-velocity measurements in the Zero velocity UPdaTe (ZUPT)-aided Inertial Navigation Systems (INS). The proposed ZUPT-aided INS using the FIBA covariance is implemented in an Adaptive Extended Kalman Filter (AEKF) framework, where the measurement covariance matrix is updated in each iteration according to the FIBA covariance. The FIBA covariance is designed to have a very high value during the swing phases in a gait cycle, and the value significantly decreases during the stance phases. As a result, the proposed method eliminates a need to use a binary stance phase detector in implementation of the ZUPT-aided INS.

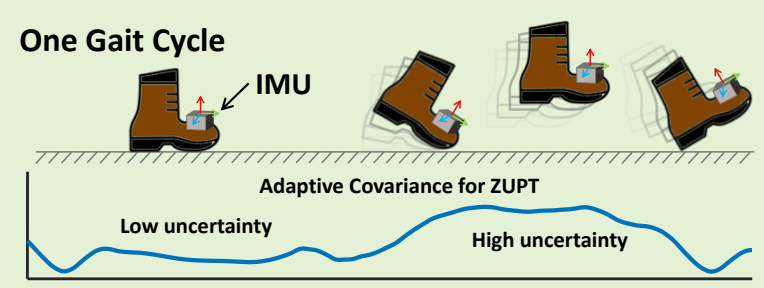

ZUPT detection

Stance phase Swing phase Two series of indoor pedestrian navigation experiments were conducted to investigate the navigation performance of the algorithm. In the first series of experiments, which included cases of walking and running, localization solutions produced by the system using the FIBA covariance demonstrated $36 \%$ and $64 \%$ improvements in navigation accuracy along the horizontal and vertical directions, respectively. In the second series of experiments, which included a pedestrian walking on different indoor terrains, such as flat planes, stairs, and ramps, the navigation accuracy of the system using the FIBA covariance reduced horizontal and vertical position errors by $12 \%$ and $45 \%$, respectively, as compared to the conventional ZUPT-aided INS.
\end{abstract}

Index Terms - Indoor Pedestrian Navigation, Inertial Measurement Unit, Zero Velocity Update, Adaptive Extended Kalman Filter, Inertial Navigation, Foot-Instability-Based Adaptive Covariance, Foot-mounted IMU

\section{INTRODUCTION}

Accurate and reliable self-contained indoor localization systems are essential for firefighters, first responders, and soldiers as the personnel often operate in environments where signals of Global Navigation Satellite System (GNSS) are degraded or unavailable, visibility is poor due to smoke, and information about surrounding Radio-Frequency (RF) infrastructures might not be accessible [1]. The latter two conditions limit the performance of many existing alternative localization systems, including 1) radio-based navigation systems utilizing Wireless Local Area Network (WLAN), Bluetooth, Long-Term Evolution (LTE), and Ultra-Wide Band (UWB) [2] and 2) vision-based navigation systems that determine position of a camera based on environmental visual features [3]. In such environments, pedestrian Inertial Navigation Systems (INS) are preferable as positioning approaches, because the systems operate in a self-contained manner, require short installation time, and provide consistently available measurements [4].

This work is performed under the following financial assistance award: 70NANB17H192 from US department of Commerce, National Institute of Standards and Technology (NIST).

C.-S. Jao and A.M. Shkel are with Microsystems Lab, Department of Mechanical and Aerospace Engineering, University of California, Irvine, CA 92697 USA, (e-mail: chishihj, ashkel@uci.edu).
Pedestrian INS utilizes Inertial Measurement Units (IMUs) mounted or attached on different parts of a human body, including on a head [5], in a pocket [6], and on a foot [7]-[9], and uses a combination of IMU measurements and local bio-mechanical information for positioning. Among these localization solutions, the systems using foot-mounted IMUs have drawn attention for their ability to significantly enhance a strapdown INS using a Zero velocity UPdaTe (ZUPT) algorithm [10]. The strapdown INS performs localization by dead reckoning based on IMU measurements [11]. Due to noise and stochastic time-varying biases of IMUs, small navigation errors build up in each dead reckoning step, and an accumulated position error of inertial navigation can exceed one meter within just a few seconds of navigation using consumer-grade IMUs [12]. The ZUPT algorithm enhances inertial navigation based on an observation that velocities of a person's foot during walking are nearly equal to zero during the stance phase of a gait cycle. Using this navigation approach, the algorithm periodically resets velocity errors of the INS when the stance phase is detected. The ZUPT-aided INS has been theoretically predicted and experimentally demonstrated to achieve an error of less than $1 \%$ of the traveling distance with an industrialgrade IMU [13] [14].

A conventional implementation of ZUPT-aided INS uses 
a stance phase detector to determine when to feedback the pseudo-zero-velocity measurements to compensate for residual velocity of the INS in an Extended Kalman Filter (EKF) framework [15]. In an ideal scenario of the ZUPT algorithm, the velocity of a foot should only be reset to zero when the foot in the stance phase is completely stationary to avoid unmodeled errors. However, the completely stationary scenario almost never happens in practice when a pedestrian is performing daily activities, such as walking, running, climbing stairs, and crawling. If the ZUPT-aided INS does not calibrate the velocity state with the zero-velocity measurements in a long period of time, the velocity error can grow unbounded, and the position estimates would quickly drift to an unacceptable range. Therefore, most existing stance phase detectors, including the conventional detectors, such as the AccelerationMoving Variance (AMV) detector, Acceleration-Magnitude (AM) detector, Angular Rate Energy (ARE) detector, and the Stance Hypothesis Optimal dEtection (SHOE) detector, as well as detectors utilizing adaptive mechanisms [16] [17], machine learning [18] [19], and sensor fusion [20]-[22], indicate the stance phase even when the foot is not completely still. Although implementations using the referenced approaches have been effective in reducing the errors of standalone INS, they introduced additional modeling errors to the navigation solutions due to a frequent violation of the assumption that the velocities are zero during the stance period [23]. The violation of the assumption misled the ZUPT algorithm to over-confidently correct velocity states with the measurement covariance matrix that had low-value entrees. The modeling error can accumulate at each step, decreasing the navigation accuracy of the ZUPT-aided INS.

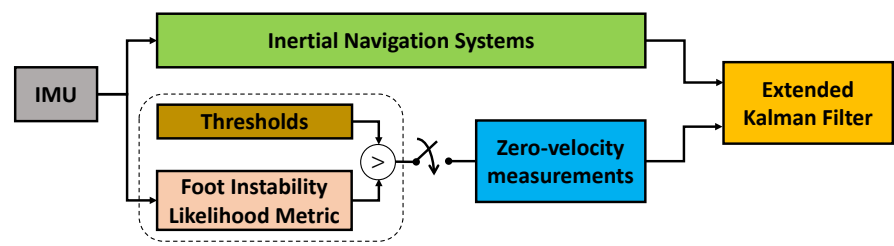

Stance Phase Detector

(a)

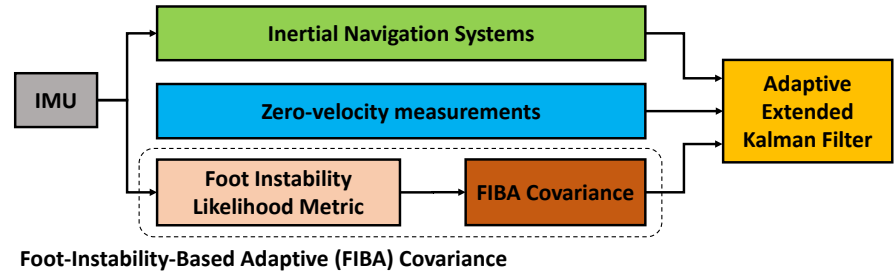

(b)

Fig. 1. (a) A conventional ZUPT-aided INS using the stance phase detector with a threshold. (b) The proposed ZUPT-aided INS implemented in an AEKF with the developed FIBA covariance. The proposed system does not use a stance phase detector and adopts a covariance matrix for the zero-velocity measurements that varies in each iteration based on the instability level of a foot.

In this paper, we propose a novel mechanism that implements a ZUPT-aided INS with a Foot-Instability-Based Adaptive (FIBA) covariance in an Adaptive Extended Kalman Filter (AEKF) framework. The proposed mechanism, which is shown in Fig. 1(b), aims to minimize one of the modeling errors in the ZUPT algorithm by using an adaptive measure- ment covariance matrix to avoid feeding back the zero-velocity measurements with high confidence when an inertial sensor mounted on a foot is not completely stationary. Compared to the conventional ZUPT-aided INS shown in Fig. 1(a), the proposed mechanism feedbacks the pseudo measurements of zero velocity at every time instance in the AEKF with the measurement covariance matrix updated by the FIBA covariance that varies according to a foot instability likelihood metrics, which is a statistics defined in this paper to quantify the instability level of the pedestrian's foot based on readings from the foot-mounted IMU. The FIBA covariance is designed to have a significantly higher value when an IMU mounted on the foot is unstable, which are the cases when the IMU experiences forces other than the gravity of the Earth. The higher value of the FIBA covariance in these cases causes the feedback of zero-velocity measurements to have a numerically minimal effect on other navigation states. When the sensor unit is stable, the FIBA covariance automatically declines to low values that are sufficient to compensate for residual velocities of a standalone INS. This property of the FIBA covariance not only reduces the modeling error of the ZUPT algorithm but also enables an alternative implementation of the ZUPTaided INS without using a stance phase detector, which could potentially reduce the computational complexity.

This paper makes the following contributions:

1) provides a foot instability likelihood metrics to quantify instability level of a foot, based on readings from inertial sensors;

2) develops a FIBA covariance that describes the uncertainty of the zero-velocity measurements in different scenarios for the ZUPT-aided INS;

3) implements the ZUPT-aided INS using the developed FIBA covariance in an AEKF framework without a stance phase detector;

4) verifies the proposed ZUPT-aided INS using the FIBA covariance with real-world indoor pedestrian navigation experiments.

The rest of the paper is organized as follows. Section II presents the proposed FIBA covariance for the ZUPTaided INS. Section II also includes the derivation of the foot instability likelihood metrics. Experimental results are presented in Section III. Finally, Section IV concludes the paper with a highlight of our main results.

\section{THE PROPOSED APPROACH}

The proposed ZUPT-aided INS using the FIBA covariance is implemented in an AEKF framework, which is illustrated by the block diagram presented in Fig. 1(b). The propagation step and the update step of the AEKF used in this paper are similar to the system discussed in [24]. However, in implementation presented in this paper, the zero-velocity measurement covariance matrix $\mathbf{R}_{\text {ZUPT }}$ is adjusted according to the proposed FIBA covariance in each update step of the AEKF.

In this section, we discuss the derivation of the foot instability likelihood metrics, the design of the proposed FIBA covariance, and parameters selection for the FIBA covariance. 


\section{A. Modeling instability of foot dynamics}

The FIBA covariance for zero-velocity measurements aims to provide a low uncertainty when a sensor unit mounted on a foot is stable, which is the case of the sensors being completely stationary, and a high uncertainty when the sensor is unstable, which is the case of the sensors experiencing a motion. The moving case and stationary case are denoted as $H_{0}$ and $H_{1}$, respectively. To model the instability level of the sensor with a metrics, this paper derives a Likelihood Ratio (LR) statistics that describes the probabilities of occurrence of any of the two cases based on IMU measurements $\mathbf{z}_{n}$. The foot instability likelihood metrics is considered to be proportional to the LR statistics.

The derivation of the LR statistics is similar to the derivation of the SHOE detector presented in [25]. The inertial sensor measurements $\mathbf{y}_{k}$ are modeled using the following notations:

$$
\mathbf{y}_{k}=\left[\begin{array}{c}
\mathbf{y}_{k}^{\alpha} \\
\mathbf{y}_{k}^{\omega}
\end{array}\right]=\left[\begin{array}{c}
\mathbf{s}_{k}^{\alpha} \\
\mathbf{s}_{k}^{\omega}
\end{array}\right]+\left[\begin{array}{c}
\mathbf{v}_{k}^{\alpha} \\
\mathbf{v}_{k}^{\omega}
\end{array}\right]=\mathbf{s}_{k}+\mathbf{v}_{k}
$$

Here, $\mathbf{s}_{k}^{\alpha} \in \mathbb{R}^{3}$ and $\mathbf{s}_{k}^{\omega} \in \mathbb{R}^{3}$ denote the IMU-experienced acceleration and angular rate, respectively. Vectors $\mathbf{v}_{k}^{\alpha} \in$ $\mathbb{R}^{3}$ and $\mathbf{v}_{k}^{\omega} \in \mathbb{R}^{3}$ represent the measurement noise of the accelerometer and gyroscope, respectively. In our derivation, we assumed that the measurement noise of accelerometers and gyroscopes has two components. One of the components is described by independent and identically-distributed white Gaussian noises. The other component is stochastic biases. We considered that the stochastic biases could be minimized by subtracting IMU measurements with bias states of the EKF used in ZUPT-aided INS. Therefore, in this derivation, the measurement noise of accelerometers and gyroscopes were modeled as white Gaussian noises with respective variances $\sigma_{\alpha}^{2}$ and $\sigma_{\omega}^{2}$.

In the case $H_{0}$, signal patterns of foot-mounted IMU measurements are unknown. In the case $H_{1}$, we hypothesize that the accelerometer experiences only the gravitational acceleration, and the angular rate experienced by the gyroscope is zero. More formally, for the two cases, we assume the sensor measurements should satisfy the following conditions:

$$
\begin{aligned}
& H_{0}: \exists k \in \Omega_{n}, \mathbf{s}_{k}^{\alpha} \neq g \mathbf{u}_{n}, \mathbf{s}_{k}^{\omega} \neq 0_{3 \times 1}, \\
& H_{1}: \forall k \in \Omega_{n}, \mathbf{s}_{k}^{\alpha}=g \mathbf{u}_{n}, \mathbf{s}_{k}^{\omega}=0_{3 \times 1},
\end{aligned}
$$

where $\mathbf{u}_{n}$ is a $3 \times 1$ unit vector, $g$ is the gravitational constant, and $\Omega_{n}=\{l \in \mathbb{N}, n \leq l<N-1\}$ is a collection of the sensor measurement indexes at time $n$ with a window of length $N$.

Following the derivation described in [25], the probability density function (pdf) of collected IMU measurements $z_{n}$ under $H_{0}$ is expressed as

$$
p\left(\mathbf{z}_{n} ; H_{0}\right)=\frac{1}{\left(2 \pi \sigma_{\alpha}^{2}\right)^{3 N / 2}\left(2 \pi \sigma_{\omega}^{2}\right)^{3 N / 2}},
$$

where $\mathbf{z}_{n}=\left\{\mathbf{y}_{k}\right\}_{k=n}^{k=N-1}$. The pdf under $H_{1}$ is given by

$$
\begin{aligned}
& p\left(\mathbf{z}_{n} ; H_{1}\right)= \\
& \frac{1}{\left(2 \pi \sigma_{\alpha}^{2}\right)^{3 N / 2}} \exp \left(-\frac{1}{2 \sigma_{\alpha}^{2}} \sum_{k \in \Omega_{n}}\left(\left\|\mathbf{y}_{k}^{\alpha}-g \frac{\overline{\mathbf{y}}_{k}^{\alpha}}{\left\|\overline{\mathbf{y}}_{k}^{\alpha}\right\|}\right\|^{2}\right)\right. \\
& \times \frac{1}{\left(2 \pi \sigma_{\omega}^{2}\right)^{3 N / 2}} \exp \left(-\frac{1}{2 \sigma_{\omega}^{2}} \sum_{k \in \Omega_{n}}\left(\left\|\mathbf{y}_{k}^{\omega}\right\|^{2}\right),\right.
\end{aligned}
$$

where

$$
\overline{\mathbf{y}}_{k}^{\alpha}=\frac{1}{N} \sum_{k \in \Omega_{n}} \mathbf{y}_{k}^{\alpha} .
$$

The LR statistics $L\left(\mathbf{z}_{n}\right)$ is derived from (1) and (2), having the following form

$$
\begin{aligned}
L\left(\mathbf{z}_{n}\right) & =\frac{p\left(\mathbf{z}_{n} ; H_{0}\right)}{p\left(\mathbf{z}_{n} ; H_{1}\right)} \\
& =\exp \left(\sum_{k \in \Omega_{n}} \frac{1}{2 \sigma_{\alpha}^{2}}\left\|\mathbf{y}_{k}^{\alpha}-g \frac{\overline{\mathbf{y}}_{k}^{\alpha}}{\left\|\overline{\mathbf{y}}_{k}^{\alpha}\right\|}\right\|^{2}+\frac{1}{2 \sigma_{\omega}^{2}}\left\|\mathbf{y}_{k}^{\omega}\right\|^{2}\right) .
\end{aligned}
$$

An example of the LR statistics profile collected in an indoor walking-and-running experiment is demonstrated in Fig. 2(a). Since the LR statistics is always positive, the foot instability likelihood metrics $S\left(\mathbf{z}_{n}\right)$ takes a scaled version of the logarithm of (3) to increase the numerical discrepancy of small values, which corresponds to the case when a foot is relatively stable. The foot instability likelihood metrics $S\left(\mathbf{z}_{n}\right)$ is expressed as follows:

$$
\begin{aligned}
S\left(\mathbf{z}_{n}\right) & =\frac{2}{N} \log \left(L\left(\mathbf{z}_{n}\right)\right) \\
& =\frac{1}{N}\left(\sum_{k \in \Omega_{n}}\left(\frac{1}{\sigma_{\alpha}^{2}}\left\|\mathbf{y}_{k}^{\alpha}-g \frac{\overline{\mathbf{y}}_{k}^{\alpha}}{\left\|\overline{\mathbf{y}}_{k}^{\alpha}\right\|}\right\|^{2}+\frac{1}{\sigma_{\omega}^{2}}\left\|\mathbf{y}_{k}^{\omega}\right\|^{2}\right)\right) .
\end{aligned}
$$

Fig. 2(b) gives an example of the foot instability likelihood metrics profile collected in the same experiment involving indoor walking and running. We can see that (4) gives a lower value in the case of the sensor being stationary than in the case of the moving sensor. Two phenomena can be noticed in Fig. 2(b). First, in the case where an IMU is traveling at a constant velocity without any rotation, the value of the foot instability likelihood metrics would be on the same level as in the case of the IMU being completely stationary. However, based on our observations in pedestrian navigation experiments, the constant velocity scenarios are unlikely to occur when the IMU is mounted on a shoe. Second, the foot instability likelihood metrics has an identical expression to the statistics metrics used by the SHOE detector. However, the statistics metrics and the foot instability likelihood metrics play two different roles. In the SHOE detector, the statistics metrics is utilized to compare with a threshold for zero-velocity event detection. In this paper, the foot instability likelihood metrics is considered as an approach to quantify the instability level of a pedestrian's foot based on inertial sensor readings, enabling the covariance matrix of zero-velocity measurements to dynamically adjust its values in different scenarios. 


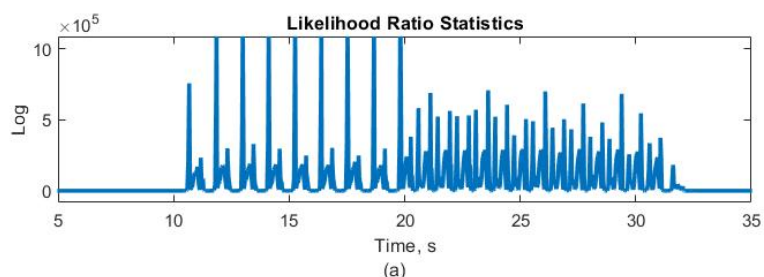

Foot Instability Likelihood Metric

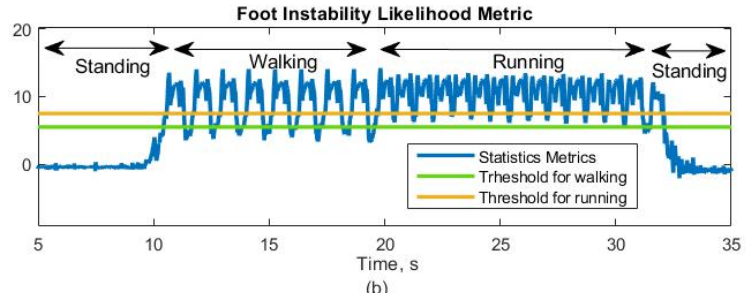

(b)

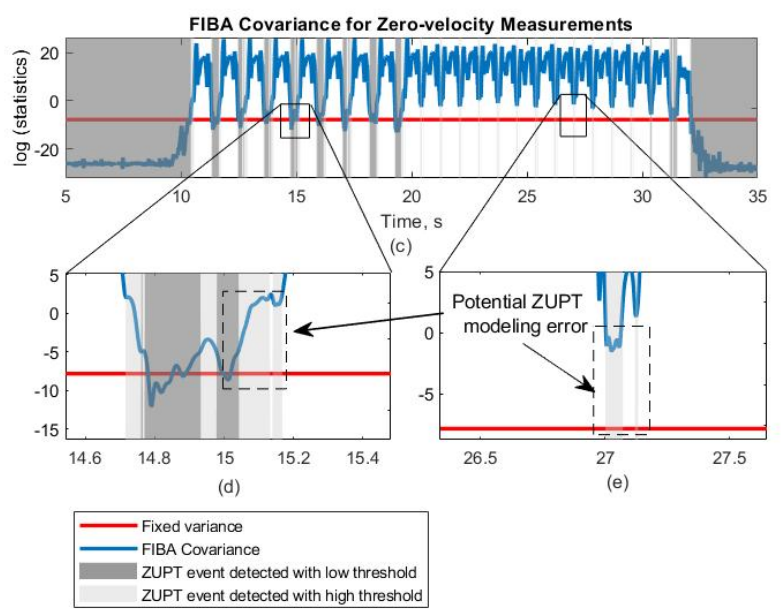

Fig. 2. (a) A profile of the Likelihood Ratio statistics, expressed in (3), in an indoor pedestrian navigation experiment discussed in Section IIC. (b) The blue curve illustrates a profile of the foot instability likelihood metrics in the same experiment, expressed in (4). The green line and the blue line represent the low threshold and the high threshold used for the SHOE detector, respectively, in the experiments discussed in Section III-A. (c) The blue curve shows a profile of the proposed FootInstability-Based Adaptive (FIBA) covariance. The red horizontal line indicates the value of $\mathbf{0 . 0 2}$. The dark gray areas indicate the stance phase detected by the SHOE detector with the threshold value specified by the green line in (b). The light gray areas mark the stance phase detected by the SHOE detector with the threshold value specified by the yellow line in (b). (d) and (e) display zoomed-in versions of (c), showing two scenarios, marked with the dashed rectangles, that a pedestrian's foot was unstable, but a stance phase was detected.

\section{B. The Foot-Instability-Based Adaptive (FIBA) Covariance}

The proposed FIBA covariance is designed to have values that are varying based on the derived foot instability metrics in Eq. (3). The derived foot instability likelihood metrics demonstrates a desired property where the metrics decreases in the stable case and increases in the unstable case. However, the values might not be suitable for selection as an uncertainty of the zero-velocity measurements. In this paper, we hypothesize that the appropriate uncertainty for zerovelocity measurements can be achieved by scaling the foot instability likelihood metrics. The FIBA covariance $\sigma_{\mathrm{FIBA}}\left(\mathbf{z}_{n}\right)$ is expressed as follows:

$$
\sigma_{\mathrm{FIBA}}\left(\mathbf{z}_{n}\right)=\beta S\left(\mathbf{z}_{n}\right)^{\gamma}, \beta \in \mathbb{R}^{+}, \gamma \in \mathbb{R}
$$

In (5), $\beta$ and $\gamma$ are hyper-parameters of the FIBA covariance that need to be selected. Note that

$$
\sigma_{\mathrm{FIBA}}\left(\mathbf{z}_{n}\right)>0, \forall \mathbf{z}_{n} .
$$

The proposed FIBA covariance $\sigma_{\mathrm{FIBA}}\left(\mathbf{z}_{n}\right)$ is used to update the covariance matrix $\mathbf{R}_{\mathrm{ZUPT}}\left(\mathbf{z}_{n}\right)$ for the zero-velocity measurements in each iteration in the AEKF framework. We assume that the zero-velocity measurements are uncorrelated and that uncertainties for the measurements along the 3-axis are identical. $\mathbf{R}_{\mathrm{ZUPT}}\left(\mathbf{z}_{n}\right)$ is presented as follows:

$$
\mathbf{R}_{\mathrm{ZUPT}}\left(\mathbf{z}_{n}\right)=\left[\begin{array}{ccc}
\sigma_{\mathrm{FIBA}}^{2}\left(\mathbf{z}_{n}\right) & 0 & 0 \\
0 & \sigma_{\mathrm{FIBA}}^{2}\left(\mathbf{z}_{n}\right) & 0 \\
0 & 0 & \sigma_{\mathrm{FIBA}}^{2}\left(\mathbf{z}_{n}\right)
\end{array}\right]
$$

The diagonal structure of $\mathbf{R}_{\mathrm{ZUPT}}\left(\mathbf{z}_{n}\right)$ and the positivity property of the term $\sigma_{\mathrm{FIBA}}^{2}\left(\mathbf{z}_{n}\right)$ guarantee $\mathbf{R}_{\mathrm{ZUPT}}\left(\mathbf{z}_{n}\right)$ to be a proper covariance matrix.

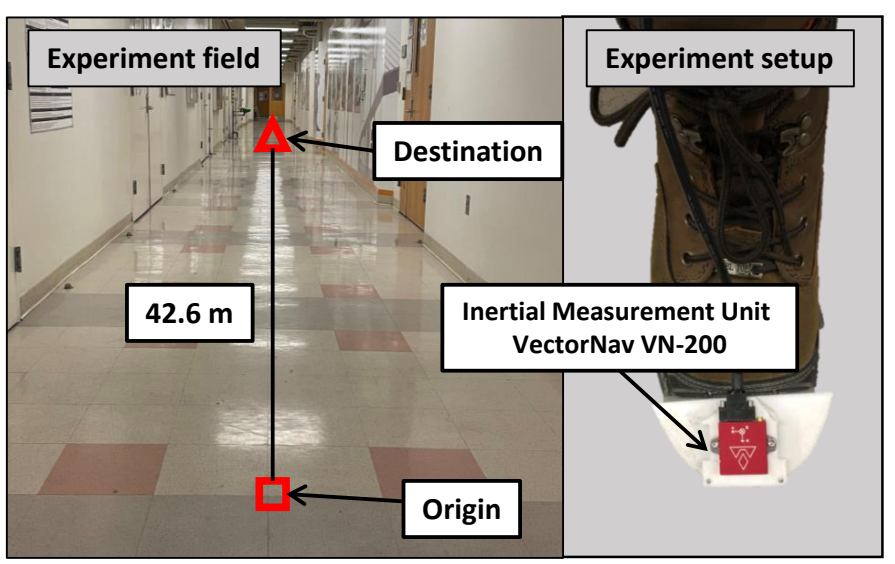

Fig. 3. The scenario and setup of the experiments discussed in Section II-C. The red square indicates the nominal starting point of the experiment, and the red triangle marks the nominal destination. The distance between the starting point and the destination was $42.6 \mathrm{~m}$, which was measured by a ruler. The IMU used in the experiments was a VectorNav VN-200 IMU. The sensor was mounted on a fixture securely attached to the toe side of the boot. The sampling rate was set to 800 $\mathrm{Hz}$.

\section{Hyper-parameter Selection}

This paper uses a data-driven approach to estimate values of the hyper-parameters $\beta$ and $\gamma$. We conducted pedestrian navigation experiments in the Engineering Gateway Building at the University of California, Irvine. The experimental setup and scenarios are shown in Fig. 3. The IMU VectorNav VN-200 was mounted on the toe side of the right shoe. In the experiments, a pedestrian first walked at a speed of approximately $60 \mathrm{step} / \mathrm{sec}$ on a straight line for $20 \mathrm{~m}$ and then ran at a speed of approximately $100 \mathrm{step} / \mathrm{sec}$ along the line for $22.6 \mathrm{~m}$. An example of the trajectory inside the building is illustrated in Fig. 4. The pedestrian repeated the same experiments 10 times. The relative ground truth location of the destination was determined by a ruler.

The selection of hyper-parameters aims to minimize navigation errors. Based on IMU measurements collected in the experiments, we implemented the ZUPT-aided INS with the proposed FIBA covariance and swept the values of $\beta$ with values $\left[e^{-15}, \quad e^{-14.5}, \quad e^{-14.0}, \quad \ldots, e^{15.0}\right]$ and $\gamma$ with values $[-3,-2.5,-2, \quad \ldots, \quad 3]$. For each pair of $\beta$ and $\gamma$ 


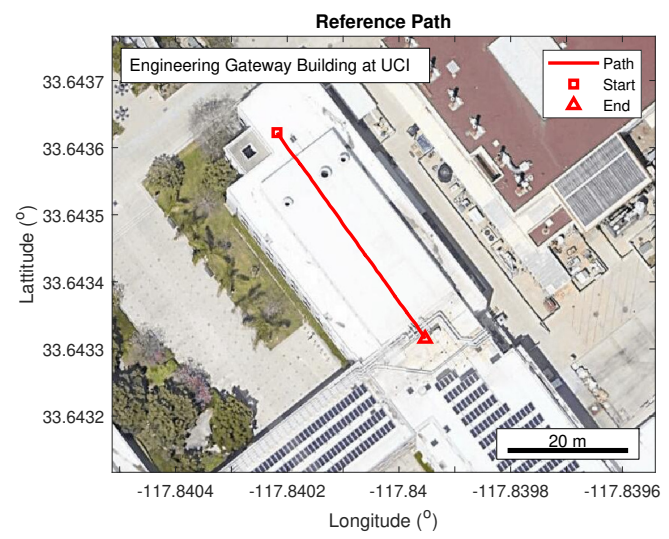

Fig. 4. A reference path for indoor pedestrian navigation experiments, described in Section II-C.

values, we calculated the Root Mean Square Errors (RMSEs) based on the loop-closure errors of the ten experiments. Fig. 5 presents the RMSEs when different values of $\beta$ and $\gamma$ were used. The hyper-parameters that corresponded to the minimum error are summarized in TABLE I.

TABLE I

HYPER-PARAMETERS FOR THE FIBA COVARIANCE

\begin{tabular}{cc}
\hline Hyper-parameter & Value \\
\hline$\beta$ & $\exp (-4.5)$ \\
$\gamma$ & 1.8 \\
\hline
\end{tabular}

\section{Discussion}

With $\beta=e^{-4.5}$ and $\gamma=1.8$, an example of profile of the FIBA covariance is illustrated in Fig. 2(c), and two zoomedin versions are shown in Fig. 2(d) and Fig. 2(e), respectively. The blue curves represent the value of the FIBA covariance, varying based on the IMU measurements collected in the experiments described in Section II-C, and the red horizontal line indicates a value of the variance of the zero-velocity measurements that are commonly used in other pedestrian navigation systems using foot-mounted IMUs. The dark and the light gray areas in 2(c) illustrate the stance phases detected by the SHOE detector with a threshold represented by the green and the yellow horizontal lines in 2(b), respectively. The following three features can be observed in Fig. 2(c), Fig. 2(d), and Fig. 2(e).

- In Fig. 2(c), the FIBA covariance during the stance phase decreases to a comparable level defined by the red line, which marked a value commonly used for the variance of zero-velocity measurements in implementation of conventional ZUPT-aided INS. The decrease indicates that the zero-velocity measurements have low uncertainty, and the velocity error would be reduced during this period. During the swing phase, the FIBA covariance increases sharply, leading to a result that the zero-velocity measurements have high uncertainty, and the velocity state in the navigation solutions would not be numerically affected by the zero-velocity feedback. This property of the proposed FIBA covariance eliminates the need to use a stance phase detector for the ZUPT-aided INS.

- In the period pointed by the black arrow in Fig. 2(d), a stance phase highlighted in light gray is detected, but the

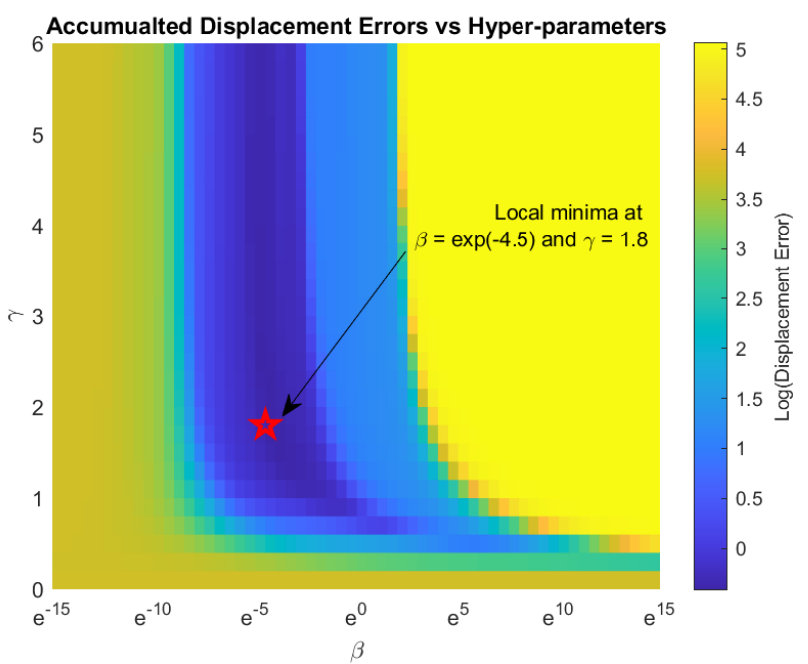

Fig. 5. The accumulated position errors at the destination estimated by the ZUPT-aided INS using the proposed FIBA covariance with different values of the hyper-parameter $\boldsymbol{\beta}$ and $\boldsymbol{\gamma}$ in the indoor pedestrian navigation experiments discussed in Section II-C. The minimum displacement error, marked with the red pentagram, occurred at $\beta=e^{-4.5}$ and $\gamma=1.8$.

instability of the shoe is higher than in other regions of the stance phase. In our opinion, the instability was due to the fact that the foot of a pedestrian could still move slightly during the stance phase, and the movement generated acceleration and angular velocity that contributed to higher values of the foot instability likelihood metrics. In conventional ZUPT-aided INS using a stance phase and a constant measurement variance, the filter could be overconfident in the zero-velocity measurements, resulting in a modeling error. With the FIBA covariance, the uncertainty was automatically tuned to a higher number, reducing the likelihood of the modeling error.

- Fig. 2(e) shows a segment of the FIBA covariance collected when the pedestrian was running. In this period, the SHOE detector with a threshold indicated by the green line in Fig. 2(b) was not able to detect the stance phase. The SHOE detector with the threshold indicated by the yellow line in Fig. 2(b) could identify a stance phase, but the usage of the constant covariance could introduce a modeling error because instability of the foot when running should be higher than in the case of walking. In this example, the value of the FIBA covariance in the running case was higher than in the walking case, indicating the modeling error is reduced when the FIBA covariance was used for the ZUPT-aided INS.

\section{EXPERIMENTAL VALIDATION}

To investigate the navigation performance of the proposed ZUPT-aided INS using the FIBA covariance, two series of experiments were conducted in the Engineering Gateway building at the University of California, Irvine. The first series investigated the navigation performance in the case of traveling at two different speeds. The second series evaluated the performance in the case of traveling on different terrains. The experimental setup used for the two series of experiments is shown in Fig. 3. A VectorNav IMU VN-200 was mounted on a customized fixture which was firmly attached to the toe 

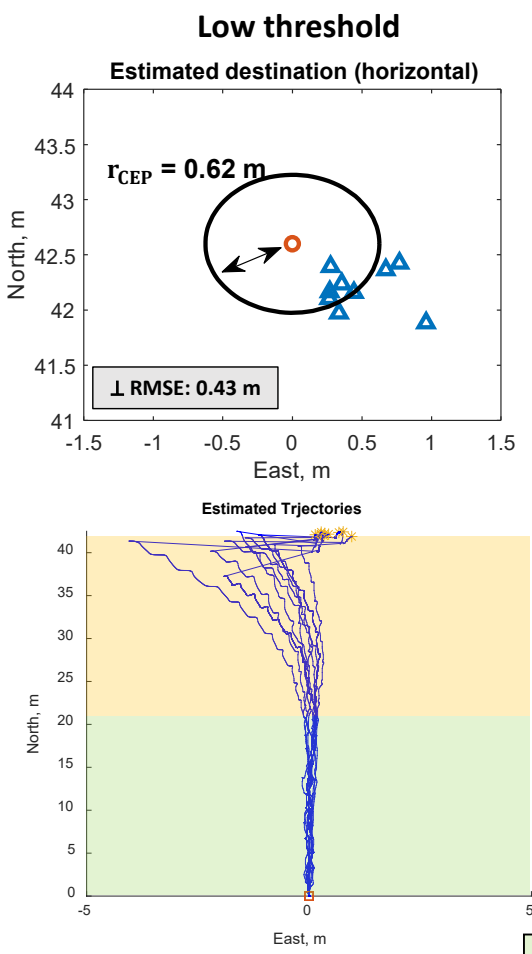

(a)
FIBA covariance
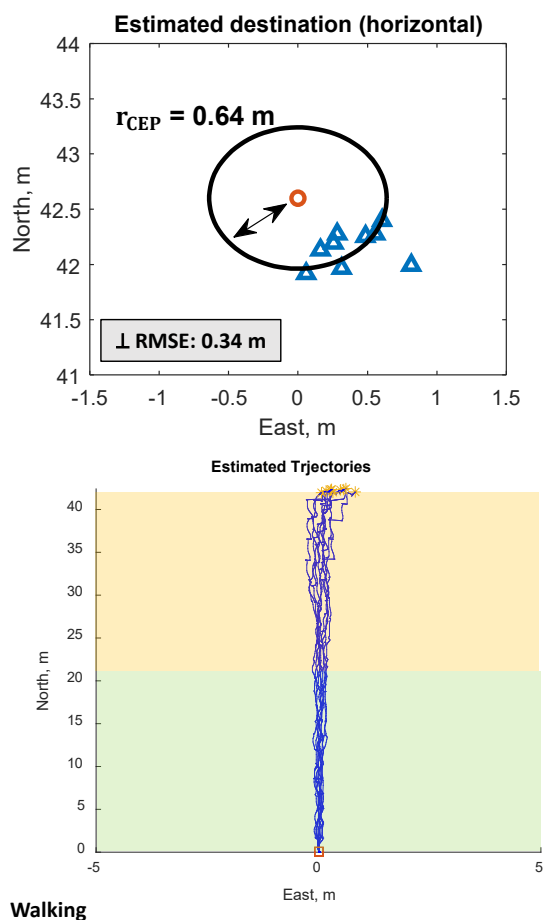

(b)
High threshold
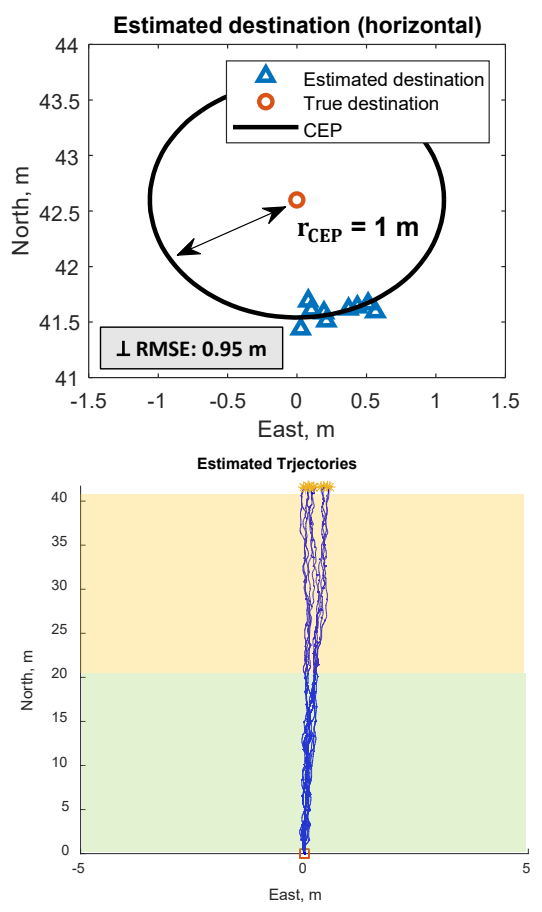

(c)

Fig. 6. (a) Trajectories and destinations estimated by the ZUPT-aided INS using the SHOE detector with a low threshold in the first series of experiments described in Section III-A. The value of the low threshold is indicated by the green line in Fig. 2(b). (b) Trajectories and destinations estimated by the ZUPT-aided INS using the proposed FIBA covariance in the same experiments. The hyper-parameters used for the FIBA covariance are summarized in TABLE I. (c) Trajectories and destinations estimated by the ZUPT-aided INS using the SHOE detector with a high threshold in the experiments. The value of the high threshold is represented by the yellow line in Fig. 2(b). In these experiments, the ZUPT-aided INS using the FIBA covariance reduced the maximum displacement errors from $4 \mathrm{~m}$ to less than $1 \mathrm{~m}$, as compared to the case using the low thresholds. When compared with solution using the high threshold, the FIBA covariance improved navigation performance by of $36 \%$ horizontally and $64 \%$ vertically.

side of the pedestrian's right shoe. The IMU was connected to a laptop held by the pedestrian for data recording. The sampling rate of the IMU was set to $800 \mathrm{~Hz}$.

\section{A. Different traveling speeds}

The first series of experiments was the same as the experiments conducted for parameter selection of the FIBA covariance, which is described in Section II-C. A reference trajectory is shown in Fig. 4. In this experiments, we evaluated the navigation accuracy of the ZUPT-aided INS using the SHOE detector with a low threshold specified by the green line in 2(b), the SHOE detector with a high threshold illustrated by the yellow line in 2(b), and the FIBA covariance. The zerovelocity variance used for the conventional ZUPT-aided INS was set to $0.02 \mathrm{~m} / \mathrm{s}$. For the horizontal displacement error, we used Circular Error Probable (CEP), which is a circle centered at the ground truth location with a radius enclosing $50 \%$ of the data. For the vertical displacement error, we calculated the RMSE based on the estimated destination.

The navigation solutions are presented in Fig. 6. It can be observed that the horizontal displacement errors of destinations estimated by solutions that used the low threshold and the FIBA covariance had similar values, which were $0.62 \mathrm{~m}$ and $0.64 \mathrm{~m}$, respectively. The horizontal CEP in the case of using the high threshold was increased to $1 \mathrm{~m}$. The vertical RMSEs in the case of the FIBA covariance was 0.34, which was smaller than the other two localization solutions. Ratios of position errors and trajectory lengths in this series of experiments are summarized in TABLE II.

\section{TABLE II}

Percentage of Position ERROR IN TRAJeCtory LENGTH FOR THE $\mathbf{1}^{\text {ST }}$ SERIES OF EXPERIMENTS

\begin{tabular}{cccc}
\hline & Low threshold & FIBA covariance & High threshold \\
\hline Horizontal & $1.46 \%$ & $1.51 \%$ & $2.35 \%$ \\
Vertical & $1.01 \%$ & $0.8 \%$ & $2.24 \%$ \\
\hline
\end{tabular}

Two observations can be made in this series of experiments. First, the ZUPT-aided INS that was using a high threshold had larger displacement errors because the high threshold led to feeding the zero velocity measurements to the EKF when the foot was not stable, resulting in additional modeling errors. The proposed ZUPT-aided INS using the FIBA covariance also feedbacked zero-velocity measurements during this period, but the modeling error was reduced because the uncertainty of the zero-velocity measurements automatically adjusted to a higher value. Second, although the displacement errors at the destinations estimated by the ZUPT-aided INS using the low threshold had errors less than one meter, it can be seen that the estimated trajectory in Fig. 6(a) drifted away when the pedestrian started to run and the maximum error was $4 \mathrm{~m}$ along the east direction. The drift was observed because the low threshold was not able to detect the stance phase, and hence no 
ZUPT algorithm was triggered during this period. In this series of experiments, the proposed ZUPT-aided INS using the FIBA covariance demonstrated an improvement in the localization accuracy, as compared to the conventional ZUPT-aided INS using the SHOE detector with a low threshold and a high threshold.

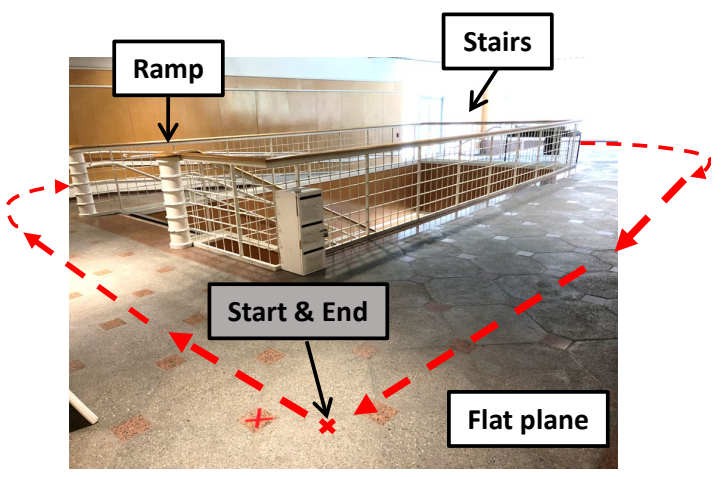

Fig. 7. The scenario of the second series of experiments discussed in Section III-B. The trajectories in the experiments included the segments of terrains of flat planes, stairs, and a ramp.

\section{B. Different terrains}

The second series of experiments investigated the navigation performance of the proposed ZUPT-aided INS using FIBA covariance when operating on different terrains. The experiments were conducted in an indoor environment illustrated in Fig. 7. In this series of experiments, a pedestrian walked a closed-loop trajectory for a length of $50 \mathrm{~m}$ in $40 \mathrm{~s}$. The path included a flat surface, a ramp, and stairs. The pedestrian repeated the same experiments 10 times

We compared the navigation performance of the proposed ZUPT-aided INS using the FIBA covariance and the conventional ZUPT-aided INS using the SHOE detector with a constant threshold. The experimental results of the two systems are shown in Fig. 8. The solution with FIBA covariance improved horizontal CEP and the vertical RMSE by $12 \%$ and $45 \%$, respectively, as compared to the conventional ZUPTaided INS. TABLE III summarizes percentage of position errors in trajectory lengths in this series of experiments. We concluded the improvement in the navigation accuracy to be a direct result of using the FIBA covariance, which reduced the modeling error in the ZUPT-aided INS. This series of experiments demonstrated that the navigation performance of the ZUPT-aided INS using the proposed FIBA covariance outperformed the case of conventional ZUPT-aided INS when traveling on terrains of flat planes, stairs, and slopes.

TABLE III

PERCENTAGE OF POSITION ERROR IN TRAJECTORY LENGTH FOR THE $2^{\text {ND }}$ SERIES OF EXPERIMENTS

\begin{tabular}{ccc}
\hline & FIBA covariance & Constant threshold \\
\hline Horizontal & $0.58 \%$ & $0.66 \%$ \\
Vertical & $0.52 \%$ & $0.96 \%$ \\
\hline
\end{tabular}

\section{CONCLUSiON}

In this paper, we introduced the FIBA covariance to dynamically adjust uncertainties of the zero-velocity measurements

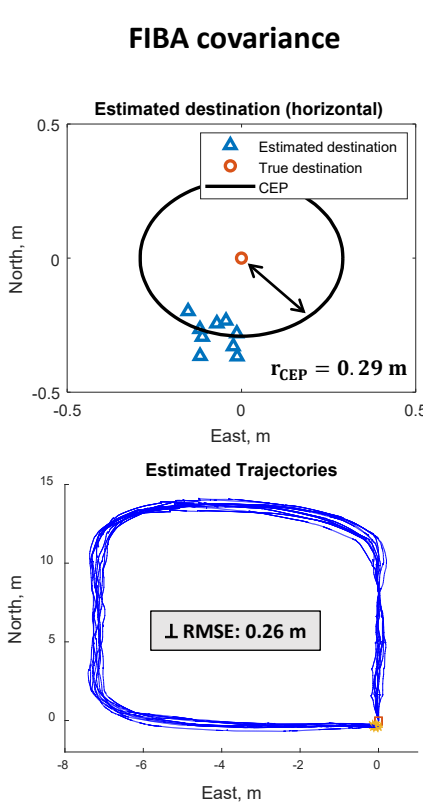

(a)
Shoe detector $\mathbf{w} /$ constant threshold
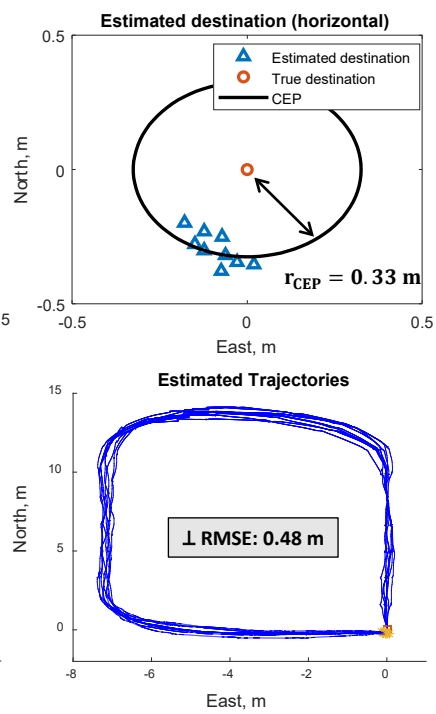

(b)
Fig. 8. (a) Trajectories and destinations estimated by the ZUPT-aided INS using the proposed FIBA covariance in the pedestrian navigation experiments described in Section III-B. The hyper-parameters used for the FIBA covariance are summarized in TABLE I. (b) Trajectories and destinations estimated by the ZUPT-aided INS using the SHOE detector with the threshold in the same experiments. The value of the threshold is indicated by the green line in Fig. 2(b). In this series of experiments, the system using the FIBA covariance outperformed the conventional ZUPT-aided INS by $\mathbf{1 2 \%}$ in terms of horizontal CEP and $\mathbf{4 5 \%}$ in terms of vertical RMSE.

in the ZUPT-aided INS, allowing to reduce the modeling error of the ZUPT algorithm. The proposed ZUPT-aided INS with the FIBA covariance was implemented in the AEKF framework, where the measurements covariance matrix for the zero-velocity measurements was updated in each iteration according to the FIBA covariance, which varied based on the instability metrics derived from foot-mounted IMU measurements. The developed FIBA covariance was demonstrated to exhibit a property that it gives a value sufficiently lower when the foot was stable, such that the zero-velocity measurements can effectively reset the velocity states. When the foot was experiencing a motion, the statistics of the FIBA covariance increased sharply, and as such the zero-velocity measurements would not have a significant numerical impact on the velocity state. This property of the FIBA covariance allows to reduce the ZUPT modeling error and eliminates the requirement to have a stance phase detector in the ZUPT-aided INS. Two series of indoor pedestrian navigation experiments were conducted in this paper to evaluate the ZUPT-aided INS using the proposed FIBA covariance. In the first series, including both walking and running activities, the solution using the FIBA covariance showed a maximum improvement in navigation accuracy of $36 \%$ horizontally and $64 \%$ vertically, as compared to the conventional ZUPT-aided INS using the SHOE detector with a constant threshold. In the second series of experiments, which included walking on different terrains of flat planes, stairs, and slopes, the ZUPT-aided INS using the FIBA covariance reduced horizontal CEP by $12 \%$ and vertical RMSE by $45 \%$, as compared to the conventional ZUPT-aided 
INS. We concluded that using the FIBA covariance in the ZUPT-aided INS can eliminate the need to use a stance phase detector and could be beneficial for navigation accuracy.

\section{ACKNOWLEDGMENT}

All analytical and numerical work was conducted in the MicroSystems Laboratory of the University of California, Irvine.

\section{REFERENCES}

[1] C. Fischer and H. Gellersen, "Location and navigation support for emergency responders: A survey," IEEE Pervasive Computing, vol. 9, no. 1 , pp. $38-47,2010$.

[2] J. Rantakokko, J. Rydell, P. Strömbäck, P. Händel, J. Callmer, D. Törnqvist, F. Gustafsson, M. Jobs, and M. Gruden, "Accurate and reliable soldier and first responder indoor positioning: multisensor systems and cooperative localization," IEEE Wireless Communications, vol. 18, no. 2, pp. 10-18, 2011.

[3] J. Fuentes-Pacheco, J. Ruiz-Ascencio, and J. M. Rendón-Mancha, "Visual simultaneous localization and mapping: a survey," Artificial Intelligence Review, vol. 43, no. 1, pp. 55-81, 2015.

[4] P. Barsocchi, S. Chessa, F. Furfari, and F. Potorti, "Evaluating ambient assisted living solutions: The localization competition," IEEE Pervasive Computing, vol. 12, no. 4, pp. 72-79, 2013.

[5] S. Beauregard, "A helmet-mounted pedestrian dead reckoning system," in International Forum on Applied Wearable Computing. Bremen, Germany: VDE, Mar. 15-16 2006.

[6] E. Munoz Diaz, "Inertial pocket navigation system: Unaided 3D positioning," Sensors, vol. 15, no. 4, pp. 9156-9178, 2015.

[7] J.-O. Nilsson, A. K. Gupta, and P. Händel, "Foot-mounted inertial navigation made easy," in International Conference on Indoor Positioning and Indoor Navigation (IPIN), Busan, Korea, Oct. 27-30, 2014.

[8] S. Askari, C.-S. Jao, Y. Wang, and A. M. Shkel, "A laboratory testbed for self-contained navigation," in IEEE International Symposium on Inertial Sensors and Systems (INERTIAL), Naples, FL, USA, Apr. 1-5, 2019.

[9] C.-S. Jao, Y. Wang, Y.-W. Lin, and A. M. Shkel, "A hybrid barometric/ultrasonic altimeter for aiding ZUPT-based inertial pedestrian navigation systems," in The International Technical Meeting of the Satellite Division of The Institute of Navigation (ION GNSS+ 2020), Virtual Conference, Sep. 21-25, 2020.

[10] A. R. Jimenez, F. Seco, C. Prieto, and J. Guevara, "A comparison of pedestrian dead-reckoning algorithms using a low-cost MEMS IMU," in IEEE International Symposium on Intelligent Signal Processing, Budapest, Hungary, Aug. 26-28, 2009.

[11] D. Titterton, J. L. Weston, and J. Weston, Strapdown inertial navigation technology. IET, 2004, vol. 17.

[12] M. Ma, Q. Song, Y. Li, and Z. Zhou, "A zero velocity intervals detection algorithm based on sensor fusion for indoor pedestrian navigation," in IEEE Information Technology, Networking, Electronic and Automation Control Conference (ITNEC), Chengdu, China, Dec. 15-17 2017.

[13] Y. Wang, C.-S. Jao, and A. M. Shkel, "Scenario-dependent ZUPT-aided pedestrian inertial navigation with sensor fusion," Journal of Gyroscopy and Navigation, vol. 12, no. 1, pp. 1-16, 2021.

[14] Y. Wang, Y.-W. Lin, S. Askari, C.-S. Jao, and A. M. Shkel, "Compensation of systematic errors in ZUPT-aided pedestrian inertial navigation," in IEEE/ION Position, Location and Navigation Symposium (PLANS), Portland, OR, USA, Apr. 20-23, 2020.

[15] C.-S. Jao, Y. Wang, and A. M. Shkel, "Pedestrian inertial navigation system augmented by vision-based foot-to-foot relative position measurements," in IEEE/ION Position, Location and Navigation Symposium (PLANS), Portland, OR, USA, Apr. 20-23, 2020.

[16] J. Wahlström, I. Skog, F. Gustafsson, A. Markham, and N. Trigoni, "Zero-velocity detection-a bayesian approach to adaptive thresholding," IEEE Sensors Letters, vol. 3, no. 6, pp. 1-4, 2019.

[17] Y. Wang and A. M. Shkel, "Adaptive threshold for zero-velocity detector in ZUPT-aided pedestrian inertial navigation," IEEE Sensors Letters, vol. 3, no. 11, pp. 1-4, 2019.

[18] B. Wagstaff, V. Peretroukhin, and J. Kelly, "Improving foot-mounted inertial navigation through real-time motion classification," in International Conference on Indoor Positioning and Indoor Navigation (IPIN), Sapporo, Japan, Sep. 18-21, 2017.
[19] Y. Wang and A. M. Shkel, "Learning-based floor-type identification in the ZUPT-aided pedestrian inertial navigation," IEEE Sensors Letters, vol. 5, no. 3, pp. 1-4, 2021.

[20] C.-S. Jao, Y. Wang, and A. M. Shkel, "A Zero Velocity Detector for Foot-mounted Inertial Navigation Systems Aided by Downward-facing Range Sensor," in IEEE Sensors Conference, Virtual Conference, Oct. 25-28, 2020.

[21] C.-S. Jao, K. Stewart, J. Conradt, E. Neftci, and A. M. Shkel, "Zero velocity detector for foot-mounted inertial navigation system assisted by a dynamic vision sensor," in DGON Inertial Sensors and Systems (ISS), Virtual Conference, Sep. 15-16, 2020.

[22] M. Ma, Q. Song, Y. Gu, Y. Li, and Z. Zhou, "An adaptive zero velocity detection algorithm based on multi-sensor fusion for a pedestrian navigation system," Sensors, vol. 18, no. 10, p. 3261, 2018.

[23] J. Wahlström and I. Skog, "Fifteen years of progress at zero velocity: A review," IEEE Sensors Journal, vol. 21, no. 2, pp. 1139-1151, 2020.

[24] Y. Wang, A. Chernyshoff, and A. M. Shkel, "Error analysis of ZUPTaided pedestrian inertial navigation," in International Conference on Indoor Positioning and Indoor Navigation (IPIN), Nantes, France, Sep. 24-27, 2018.

[25] I. Skog, P. Handel, J.-O. Nilsson, and J. Rantakokko, "Zero-velocity detection-an algorithm evaluation," IEEE Transactions On Biomedical Engineering, vol. 57, no. 11, pp. 2657-2666, 2010.

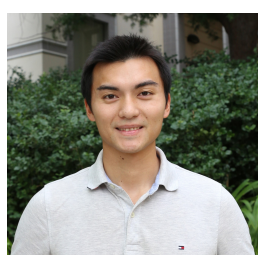

Chi-Shih Jao (S'20) received the B.S. degree in Electrical Engineering from National Tsing Hua University, Hsinchu, Taiwan, in 2015, and the M.S. degree in Electrical Engineering from Pennsylvania State University, University Park, in 2018. He is currently pursuing the Ph.D. degree with the Microsystems Laboratory, Department of Mechanical and Aerospace Engineering, University of California, Irvine, CA. His research interests include aided inertial navigation by sensor fusion approach and vision-based inertial navigation. He is a recipient of the 2019-2020 Holmes Fellowship.

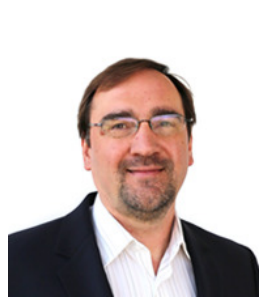

Andrei M. Shkel (Fellow, IEEE) received the diploma degree (Hons.) in mechanics and mathematics from Lomonosov's Moscow State University, Moscow, Russia, in 1991, and the Ph.D. degree in mechanical engineering from the University of Wisconsin-Madison, Madison, WI, USA, in 1997. In 2000, he joined the Faculty of the University of California, Irvine, Irvine, CA, USA, where he is currently a Professor with the Department of Mechanical and Aerospace Engineering, with a joint appointment with the Department of Electrical Engineering and Computer Science, and the Department of Biomedical Engineering. He has served as a Program Manager for the Microsystems Technology Office, Defense Advanced Research Projects Agency (DARPA), Arlington, VA, USA, from 2009 to 2013. His professional interests, reflected in over 270 publications, include solid-state sensors and actuators, micro- electromechanical systems-based neuroprosthetics, sensor-based intelligence, and control theory. He holds over 42 issued patents. His current research interests include the design, manufacturing, and advanced control of high-precision micromachined gyroscopes and self-contained inertial navigation systems. He has been the President of the IEEE Sensors Council since 2020. He was a recipient of the 2002 George E. Brown, Jr., Award, the 2005 NSF CAREER Award, the 2006 UCI HSSoE Best Faculty Research Award, the 2009 IEEE Sensors Council Technical Achievement Award, and the 2020 UCI HSSoE Innovator of the Year Award. In 2013, he received the Office of the Secretary of Defense Medal for Exceptional Public Service. He has also served on a number of editorial boards, most recently, as an Editor for the IEEE/ASME JOURNAL OF MICROELECTROMECHANICAL SYSTEMS, the Journal of Gyroscopy and Navigation, and the Founding Chair for the IEEE International Symposium on Inertial Sensors and Systems. 